\title{
Multiproduct Supply Chain Horizontal Network Integration: Models, Theory, and Computational Results
}

\author{
Anna Nagurney, Trisha Woolley, and Qiang Qiang \\ Department of Finance and Operations Management \\ Isenberg School of Management \\ University of Massachusetts \\ Amherst, Massachusetts 01003
}

July 2008; revised March 2009

Appears in the International Transactions in Operational Research (2010) 17, 333-349.

\begin{abstract}
In this paper, we develop multiproduct supply chain network models with explicit capacities, prior to and post their horizontal integration. In addition, we propose a measure, which allows one to quantify and assess, from a supply chain network perspective, the synergy benefits associated with the integration of multiproduct firms through mergers/acquisitions. We utilize a system-optimization perspective for the model development and provide the variational inequality formulations, which are then utilized to propose a computational procedure which fully exploits the underlying network structure. We illustrate the theoretical and computational framework with numerical examples.

This paper is a contribution to the literatures of supply chain integration and mergers and acquisitions.
\end{abstract}

Key words: multiproduct supply chains, horizontal integration, mergers and acquisitions, total cost minimization, synergy 


\section{Introduction}

Today, supply chains are more extended and complex than ever before. At the same time, the current competitive economic environment requires that firms operate efficiently, which has spurred interest among researchers as well as practitioners to determine how to utilize supply chains more effectively and efficiently.

In this increasingly competitive economic environment, there is also a pronounced amount of merger activity. Indeed, according to Thomson Financial, in the first nine months of 2007 alone, worldwide merger activity hit $\$ 3.6$ trillion, surpassing the total from all of 2006 combined (Wong (2007)). Interestingly, Langabeer and Seifert (2003) showed a compelling and direct correlation between the level of success of the merged companies and how effectively the supply chains of the merged companies are integrated. However, a survey of 600 executives involved in their companies' mergers and acquisitions (M\&A), conducted by Accenture and the Economist Unit (EIU), found that less than half (45\%) achieved expected cost-savings synergies (Byrne (2007)). It is, therefore, worthwhile to develop tools that can better predict the associated strategic gains associated with supply chain network integration, in the context of mergers/acquisitions, which may include, among others, possible cost savings (Eccles et al. (1999)).

Furthermore, although there are numerous articles discussing multi-echelon supply chains, the majority deal with a homogeneous product (see, for example, Dong et al. (2004), Nagurney (2006a), and Wang et al. (2007)). Firms are seeing the need to spread their investment risk by building multiproduct supply facilities, which also gives the advantage of flexibility to meet changing market demands. According to a study of the US supply output at the firmproduct level between 1972 and 1997, on the average, two-thirds of US supply firms altered their mix of products every five years (Bernard et al. (2006)). By running a multi-use plant, costs of supply may be divided among different products, which may increase efficiencies.

Moreover, it is interesting to note the relationships between merger activity to multiproduct output. For example, according to a study of the US supply output at the firm-product level between 1972 and 1997, less than 1 percent of a firm's product additions occurred due to mergers/acquisitions. Actually, 95 percent of firms, engaging in M\&A, were found to adjust their product mix, which can be associated with ownership changes (Bernard et al. (2006)). The importance of the decision as to what to offer (e.g., products and services), as well as the ability of firms to realize synergistic opportunities of the proposed merger, if any, can add tremendous value. It should be noted that a successful merger depends on the ability to measure the anticipated synergy of the proposed merger (cf. Chang (1988)). 
This paper is built on the recent work of Nagurney (2009) who developed a systemoptimization perspective for supply chain network integration in the case of horizontal mergers/acquisitions. In this paper, we also focus on the case of horizontal mergers (or acquisitions) and we extend the contributions in Nagurney (2009) to the much more general and richer setting of multiple product supply chains. Our approach is most closely related to that of Dafermos (1973) who proposed transportation network models with multiple modes/classes of transportation. In particular, we develop a system-optimization approach to the modeling of multiproduct supply chains and their integration and we explicitly introduce capacities on the various economic activity links associated with manufacturing/production, storage, and distribution. Moreover, in this paper, we analyze the synergy effects associated with horizontal multiproduct supply chain network integration, in terms of the operational synergy, that is, the reduction, if any, in the cost of production, storage, and distribution. Finally, the proposed computational procedure fully exploits the underlying network structure of the supply chain optimization problems both pre and post-integration.

We note that Min and Zhou (2002) provided a synopsis of supply chain modeling and the importance of planning, designing, and controlling the supply chain as a whole. Nagurney (2006b), subsequently, proved that supply chain network equilibrium problems, in which there is cooperation between tiers, but competition among decision-makers within a tier, can be reformulated and solved as transportation network equilibrium problems. Cheng and Wu (2006) proposed a multiproduct, and multicriterion, supply-demand network equilibrium model. Davis and Wilson (2006), in turn, studied differentiated product competition in an equilibrium framework. Mixed integer linear programming models have been used to study synergy in supply chains, which has been considered by Soylu et al. (2006), who focused on energy systems, and by $\mathrm{Xu}(2007)$.

This paper is organized as follows. The pre-integration multiproduct supply chain network model is developed in Section 2. Section 2 also introduces the horizontally merged (or integrated) multiproduct supply chain model. The method of quantification of the synergistic gains, if any, is provided in Section 3, along with new theoretical results. In Section 4 we present numerical examples, which not only illustrate the richness of the framework proposed in this paper, but which also demonstrate quantitatively how the costs associated with horizontal integration affect the possible synergies. We conclude the paper with Section 5, in which we summarize the results and present suggestions for future research. 


\section{Firm $A$}

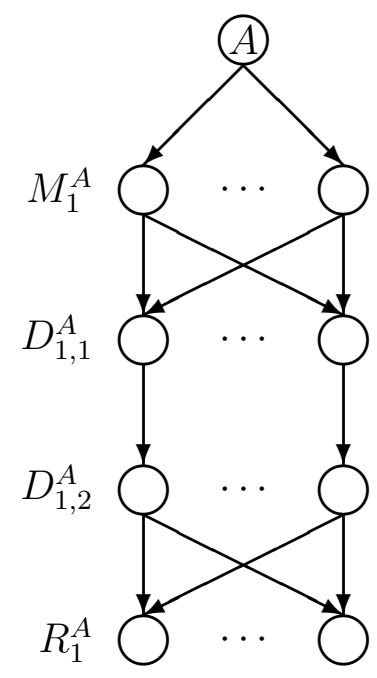

Firm $B$

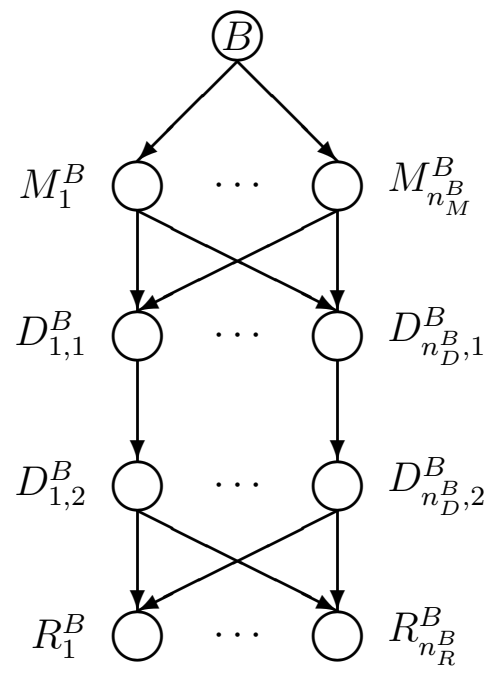

Figure 1: Supply Chains of Firms $A$ and $B$ Prior to the Integration

\section{The Pre- and Post-Integration Multiproduct Supply Chain Network Models}

This Section develops the pre- and post-integration supply chain network multiproduct models using a system-optimization approach (based on the Dafermos (1973) multiclass model) but with the inclusion of explicit capacities on the various links. Moreover, here, we provide a variational inequality formulation of multiproduct supply chains and their integration, which enables a computational approach which fully exploits the underlying network structure. We also identify the supply chain network structures both pre and post the merger and construct a synergy measure.

Section 2.1 describes the underlying pre-integration supply chain network associated with an individual firm and its respective economic activities of manufacturing, storage, distribution, and retailing. Section 2.2 develops the post-integration model. The models are extensions of the Nagurney (2009) models to the more complex, and richer, multiproduct domain.

\subsection{The Pre-Integration Multiproduct Supply Chain Network Model}

We first formulate the pre-integration multiproduct decision-making optimization problem faced by firms $A$ and $B$ and we refer to this model as Case 0 . We assume that each firm is represented as a network of its supply chain activities, as depicted in Figure 1. Each firm $i ; i=A, B$, has $n_{M}^{i}$ manufacturing facilities; $n_{D}^{i}$ distribution centers, and serves $n_{R}^{i}$ retail outlets. Let $G_{i}=\left[N_{i}, L_{i}\right]$ denote the graph consisting of nodes $\left[N_{i}\right]$ and directed links $\left[L_{i}\right]$ representing the supply chain activities associated with each firm $i ; i=A, B$. Let $L^{0}$ denote 
the links: $L_{A} \cup L_{B}$ as in Figure 1. We assume that each firm is involved in the production, storage, and distribution of $J$ products, with a typical product denoted by $j$.

The links from the top-tiered nodes $i ; i=A, B$ in each network in Figure 1 are connected to the manufacturing nodes of the respective firm $i$, which are denoted, respectively, by: $M_{1}^{i}, \ldots, M_{n_{M}^{i}}^{i}$. These links represent the manufacturing links. The links from the manufacturing nodes, in turn, are connected to the distribution center nodes of each firm $i$; $i=A, B$, which are denoted by $D_{1,1}^{i}, \ldots, D_{n_{D^{i}, 1}}^{i}$. These links correspond to the shipment links between the manufacturing facilities and the distribution centers where the products are stored. The links joining nodes $D_{1,1}^{i}, \ldots, D_{n_{D}^{i}, 1}^{i}$ with nodes $D_{1,2}^{i}, \ldots, D_{n_{D}^{i}, 2}^{i}$ for $i=A, B$, correspond to the storage links for the products. Finally, there are shipment links joining the nodes $D_{1,2}^{i}, \ldots, D_{n_{D}^{i}, 2}^{i}$ for $i=A, B$ with the retail nodes: $R_{1}^{i}, \ldots, R_{n_{R}^{i}}^{i}$ for each firm $i=A, B$. Each firm $i$, for simplicity, and, without loss of generality, is assumed to have its own individual retail outlets for delivery of the products, as depicted in Figure 1, prior to the integration.

The demands for the products are assumed as given and are associated with each product, and each firm and retail pair. Let $d_{R_{k}^{i}}^{j}$ denote the demand for product $j ; j=1, \ldots, J$, at retail outlet $R_{k}^{i}$ associated with firm $i ; i=A, B ; k=1, \ldots, n_{R}^{i}$. A path consists of a sequence of links originating at a node $i ; i=A, B$ and denotes supply chain activities comprising manufacturing, storage, and distribution of the products to the retail nodes. Let $x_{p}^{j}$ denote the nonnegative flow of product $j$, on path $p$. Let $P_{R_{k}^{i}}^{0}$ denote the set of all paths joining an origin node $i$ with (destination) retail node $R_{k}^{i}$. Clearly, since we are first considering the two firms prior to any integration, the paths associated with a given firm have no links in common with paths of the other firm. This changes (see also Nagurney (2009)) when the integration occurs, in which case the number of paths and the sets of paths also change, as do the number of links and the sets of links, as described in Section 2.2. The following conservation of flow equations must hold for each firm $i$, each product $j$, and each retail outlet $R_{k}^{i}$ :

$$
\sum_{p \in P_{R_{k}^{i}}^{0}} x_{p}^{j}=d_{R_{k}^{i}}^{j}, \quad i=A, B ; \quad j=1, \ldots, J ; \quad k=1, \ldots, n_{R}^{i},
$$

that is, the demand for each product must be satisfied at each retail outlet.

Links are denoted by $a, b$, etc. Let $f_{a}^{j}$ denote the flow of product $j$ on link $a$. We must have the following conservation of flow equations satisfied:

$$
f_{a}^{j}=\sum_{p \in P^{0}} x_{p}^{j} \delta_{a p}, \quad j=1 \ldots, J ; \quad \forall a \in L^{0},
$$

where $\delta_{a p}=1$ if link $a$ is contained in path $p$ and $\delta_{a p}=0$, otherwise. Here $P^{0}$ denotes 
the set of all paths in Figure 1, that is, $P^{0}=\cup_{i=A, B ; k=1, \ldots, n_{R}^{i}} P_{R_{k}^{i}}^{0}$. The path flows must be nonnegative, that is,

$$
x_{p}^{j} \geq 0, \quad j=1, \ldots, J ; \quad \forall p \in P^{0} .
$$

We group the path flows into the vector $x$.

Note that the different products flow on the supply chain networks depicted in Figure 1 and share resources with one another. To capture the costs, we proceed as follows. There is a total cost associated with each product $j ; j=1, \ldots, J$, and each link (cf. Figure 1) of the network corresponding to each firm $i ; i=A, B$. We denote the total cost on a link $a$ associated with product $j$ by $\hat{c}_{a}^{j}$. The total cost of a link associated with a product, be it a manufacturing link, a shipment/distribution link, or a storage link is assumed to be a function of the flow of all the products on the link; see, for example, Dafermos (1973). Hence, we have that

$$
\hat{c}_{a}^{j}=\hat{c}_{a}^{j}\left(f_{a}^{1}, \ldots, f_{a}^{J}\right), \quad j=1, \ldots, J ; \quad \forall a \in L^{0} .
$$

The top tier links in Figure 1 have total cost functions associated with them that capture the manufacturing costs of the products; the second tier links have multiproduct total cost functions associated with them that correspond to the total costs associated with the subsequent distribution/shipment to the storage facilities, and the third tier links, since they are the storage links, have associated with them multiproduct total cost functions that correspond to storage. Finally, the bottom-tiered links, since they correspond to the shipment links to the retailers, have total cost functions associated with them that capture the costs of shipment of the products.

We assume that the total cost function for each product on each link is convex, continuously differentiable, and has a bounded third order partial derivative. Since the firms' supply chain networks, pre-integration, have no links in common (cf. Figure 1), their individual cost minimization problems can be formulated jointly as follows:

$$
\text { Minimize } \sum_{j=1}^{J} \sum_{a \in L^{0}} \hat{c}_{a}^{j}\left(f_{a}^{1}, \ldots, f_{a}^{J}\right)
$$

subject to: constraints (1) - (3) and the following capacity constraints:

$$
\sum_{j=1}^{J} \alpha_{j} f_{a}^{j} \leq u_{a}, \quad \forall a \in L^{0} .
$$

The term $\alpha_{j}$ denotes the volume taken up by product $j$, whereas $u_{a}$ denotes the nonnegative capacity of link $a$. 
Observe that this problem is, as is well-known in the transportation literature (cf. Beckmann, McGuire, and Winsten (1956), Dafermos and Sparrow (1969), and Dafermos (1973)), a system-optimization problem but in capacitated form. Under the above imposed assumptions, the optimization problem is a convex optimization problem. If we further assume that the feasible set underlying the problem represented by the constraints (1) - (3) and (6) is non-empty, then it follows from the standard theory of nonlinear programming (cf. Bazaraa, Sherali, and Shetty (1993)) that an optimal solution exists.

Let $\mathcal{K}^{0}$ denote the set where $\mathcal{K}^{0} \equiv\{f \mid \exists x$ such that $(1)-(3)$ and (6) hold $\}$, where $f$ is the vector of link flows. We assume that the feasible set $\mathcal{K}^{0}$ is non-empty. We associate the Lagrange multiplier $\beta_{a}$ with constraint (6) for each $a \in L^{0}$. We denote the associated optimal Lagrange multiplier by $\beta_{a}^{*}$. This term may be interpreted as the price or value of an additional unit of capacity on link $a$; it is also sometimes refered to as the shadow price. We now provide the variational inequality formulation of the problem. For convenience, and since we are considering Case 0 , we denote the solution of variational inequality (7) below as $\left(f^{0 *}, \beta^{0 *}\right)$ and we refer to the corresponding vectors of variables with superscripts of 0 .

\section{Theorem 1}

The vector of link flows $f^{0 *} \in \mathcal{K}^{0}$ is an optimal solution to the pre-integration problem if and only if it satisfies the following variational inequality problem with the vector of nonnegative Lagrange multipliers $\beta^{0 *}$ :

$$
\begin{gathered}
\sum_{j=1}^{J} \sum_{l=1}^{J} \sum_{a \in L^{0}}\left[\frac{\partial \hat{c}_{a}^{l}\left(f_{a}^{1 *}, \ldots, f_{a}^{J *}\right)}{\partial f_{a}^{j}}+\alpha_{j} \beta_{a}^{*}\right] \times\left[f_{a}^{j}-f_{a}^{j *}\right]+\sum_{a \in L^{0}}\left[u_{a}-\sum_{j=1}^{J} \alpha_{j} f_{a}^{j *}\right] \times\left[\beta_{a}-\beta_{a}^{*}\right] \geq 0 \\
\forall f^{0} \in \mathcal{K}^{0}, \forall \beta^{0} \geq 0
\end{gathered}
$$

Proof: See Bertsekas and Tsitsiklis (1989) and Nagurney (1999).

\subsection{The Post-Integration Multiproduct Supply Chain Network Model}

We now formulate the post-integration case, referred to as Case 1. Figure 2 depicts the post-integration supply chain network topology. Note that there is now a supersource node 0 which represents the integration of the firms in terms of their supply chain networks with additional links joining node 0 to nodes $A$ and $B$, respectively.

As in the pre-integration case, the post-integration optimization problem is also concerned with total cost minimization. Specifically, we retain the nodes and links associated with the network depicted in Figure 1 but now we add the additional links connecting the 


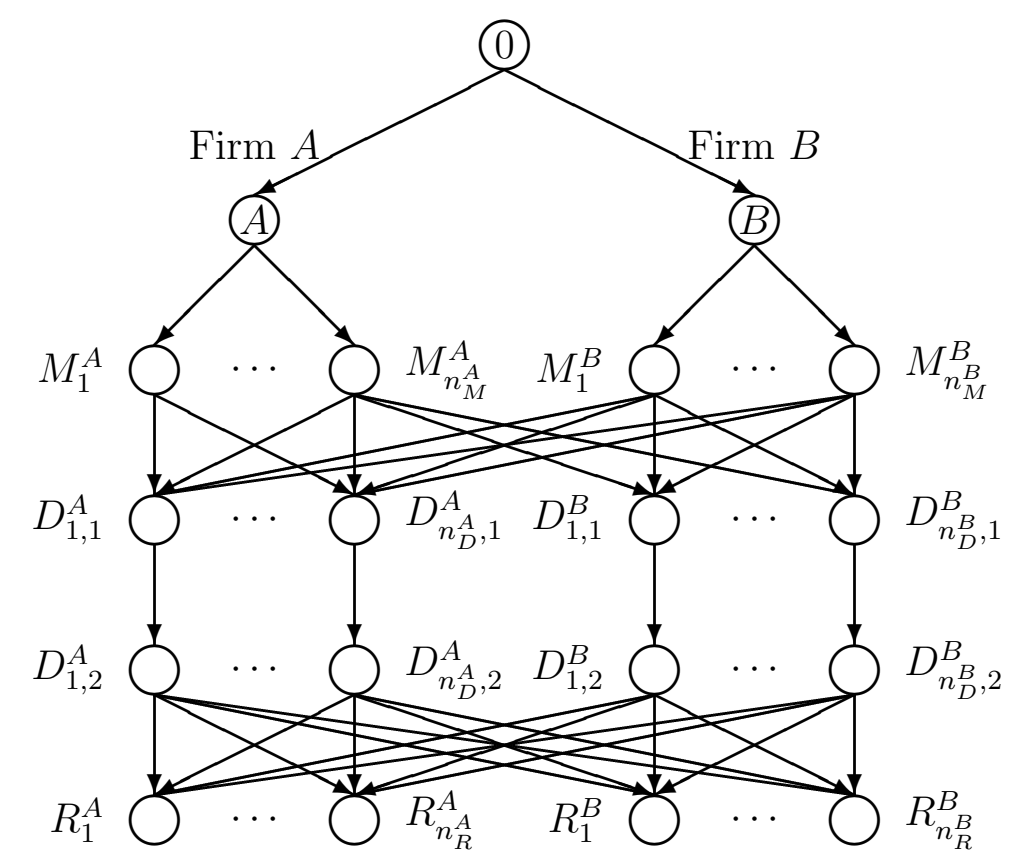

Figure 2: Supply Chain Network after Firms $A$ and $B$ Merge

manufacturing facilities of each firm and the distribution centers of the other firm as well as the links connecting the distribution centersof each firm and the retail outlets of the other firm. We refer to the network in Figure 2, underlying this integration, as $G^{1}=\left[N^{1}, L^{1}\right]$ where $N^{1} \equiv N^{0} \cup$ node 0 and $L^{1} \equiv L^{0} \cup$ the additional links as in Figure 2. We associate total cost functions as in (4) with the new links, for each product $j$. Note that if the total cost functions associated with the integration/merger links connecting node 0 to node $A$ and node 0 to node $B$ are set equal to zero, this means that the supply chain integration is costless in terms of the supply chain integration/merger of the two firms. Of course, non-zero total cost functions associated with these links may be utilized to also capture the risk associated with the integration. We will explore such issues numerically in Section 4.

A path $p$ now (cf. Figure 2) originates at the node 0 and is destined for one of the bottom retail nodes. Let $x_{p}^{j}$, in the post-integrated network configuration given in Figure 2, denote the flow of product $j$ on path $p$ joining (origin) node 0 with a (destination) retail node. Then, the following conservation of flow equations must hold:

$$
\sum_{p \in P_{R_{k}^{i}}^{1}} x_{p}^{j}=d_{R_{k}^{i}}^{j}, \quad i=A, B ; \quad j=1, \ldots, J ; \quad k=1, \ldots, n_{R}^{i},
$$

where $P_{R_{k}^{i}}^{1}$ denotes the set of paths connecting node 0 with retail node $R_{k}^{i}$ in Figure 2 . Due to the integration, the retail outlets can obtain each product $j$ from any manufacturing facility, and any distributor. The set of paths $P^{1} \equiv \cup_{i=A, B ; k=1, \ldots, n_{R}^{i}} P_{R_{k}^{i}}^{1}$.

In addition, as before, let $f_{a}^{j}$ denote the flow of product $j$ on link $a$. Hence, we must also 
have the following conservation of flow equations satisfied:

$$
f_{a}^{j}=\sum_{p \in P^{1}} x_{p}^{j} \delta_{a p}, \quad j=1, \ldots, J ; \quad \forall a \in L^{1}
$$

Of course, we also have that the path flows must be nonnegative for each product $j$, that is,

$$
x_{p}^{j} \geq 0, \quad j=1, \ldots, J ; \quad \forall p \in P^{1} .
$$

We assume, again, that the supply chain network activities have nonnegative capacities, denoted as $u_{a}, \forall a \in L^{1}$, with $\alpha_{j}$ representing the volume factor for product $j$. Hence, the following constraints must be satisfied:

$$
\sum_{j=1}^{J} \alpha_{j} f_{a}^{j} \leq u_{a}, \quad \forall a \in L^{1} .
$$

Consequently, the optimization problem for the integrated supply chain network is:

$$
\text { Minimize } \sum_{j=1}^{J} \sum_{a \in L^{1}} \hat{c}_{a}^{j}\left(f_{a}^{1}, \ldots, f_{a}^{J}\right)
$$

subject to constraints: (8) - (11).

The solution to the optimization problem (12) subject to constraints (8) through (11) can also be obtained as a solution to a variational inequality problem akin to (7) where now $a \in$ $L^{1}$. The vectors $f$ and $\beta$ have identical definitions as before, but are re-dimensioned/expanded accordingly and superscripted with a 1 . Finally, instead of the feasible set $\mathcal{K}^{0}$ we now have $\mathcal{K}^{1} \equiv\{f \mid \exists x$ such that $(8)-(11)$ hold $\}$. We assume that $\mathcal{K}^{1}$ is non-empty. We denote the solution to the variational inequality problem (13) below governing Case 1 by $\left(f^{1 *}, \beta^{1 *}\right)$ and denote the vectors of corresponding variables as $\left(f^{1}, \beta^{1}\right)$. We now, for completeness, provide the variational inequality formulation of the Case 1 problem. The proof is immediate.

\section{Theorem 2}

The vector of link flows $f^{1 *} \in \mathcal{K}^{1}$ is an optimal solution to the post-integration problem if and only if it satisfies the following variational inequality problem with the vector of nonnegative Lagrange multipliers $\beta^{1 *}$ :

$$
\begin{gathered}
\sum_{j=1}^{J} \sum_{l=1}^{J} \sum_{a \in L^{1}}\left[\frac{\partial \hat{c}_{a}^{l}\left(f_{a}^{1 *}, \ldots, f_{a}^{J *}\right)}{\partial f_{a}^{j}}+\alpha_{j} \beta_{a}^{*}\right] \times\left[f_{a}^{j}-f_{a}^{j *}\right]+\sum_{a \in L^{1}}\left[u_{a}-\sum_{j=1}^{J} \alpha_{j} f_{a}^{j *}\right] \times\left[\beta_{a}-\beta_{a}^{*}\right] \geq 0 \\
\forall f^{1} \in \mathcal{K}^{1}, \forall \beta^{1} \geq 0
\end{gathered}
$$


We let $T C^{0}$ denote the total cost, $\sum_{j=1}^{J} \sum_{a \in L^{0}} \hat{c}_{a}^{j}\left(f_{a}^{1}, \ldots, f_{a}^{J}\right)$, evaluated under the solution $f^{0 *}$ to $(7)$ and we let $T C^{1}, \sum_{j=1}^{J} \sum_{a \in L^{1}} \hat{c}_{a}^{j}\left(f_{a}^{1}, \ldots, f_{a}^{J}\right)$ denote the total cost evaluated under the solution $f^{1 *}$ to (13). Due to the similarity of variational inequalities (7) and (13) the same computational procedure can be utilized to compute the solutions. Indeed, we utilize the variational inequality formulations of the respective pre- and post-integration supply chain network problems since we can then exploit the simplicity of the underlying feasible sets $\mathcal{K}^{0}$ and $\mathcal{K}^{1}$ which include constraints with a network structure identical to that underlying multimodal system-optimized transportation network problems.

It is worthwhile to distinguish the multiproduct supply chain network models developed above from the single product models in Nagurney (2009). First, we note that the total cost functions in the objective functions (5) and (12) are not separable as they were, respectively, in the single product models in Nagurney (2009). In addition, since we are dealing now with multiple products, which can be of different physical dimensions, the corresponding capacity constraints (cf. (6) and (11)) are also more complex than was the case for their single product counterparts. We also emphasize that the above multiproduct framework contains, as a special case, the merger of firms that produce (pre-merger) distinct products, which is captured by assigning a demand of zero to those products at the respective demand markets. Of course, in such a case, the total cost functions would also be adapted accordingly.

Finally, the multiproduct models developed in this paper allow for non-zero total costs associated with the top-most merger links (cf. Figure 2), which join node 0 to nodes $A$ and $B$. In Nagurney (2009) it was assume that the corresponding total costs, in the single product case, were zero. Of course, it would also be interesting to explore the issue of "retooling" a manufacturing facility, post-merger, for it to be able to produce the other firm's product(s) in its original manufacturing facilities.

\section{Quantifying Synergy Associated with Multiproduct Supply Chain Network Integration}

We measure the synergy by analyzing the total costs prior to and post the supply chain network integration (cf. Eccles et al. (1999) and Nagurney (2009)). For example, the synergy based on total costs and proposed by Nagurney (2009), but now in a multiproduct context, which we denote here by $\mathcal{S}^{T C}$, can be calculated as the percentage difference between the total cost pre $v s$ the total cost post the integration:

$$
\mathcal{S}^{T C} \equiv\left[\frac{T C^{0}-T C^{1}}{T C^{0}}\right] \times 100 \% \text {. }
$$

From (14), one can see that the lower the total cost $T C^{1}$, the higher the synergy associated 
with the supply chain network integration. Of course, in specific firm operations one may wish to evaluate the integration of supply chain networks with only a subset of the links joining the original two supply chain networks. In that case, Figure 2 would be modified accordingly and the synergy as in (14) computed with $T C^{1}$ corresponding to that new supply chain network topology.

We now provide a theorem which shows that if the total costs associated with the integration of the supply chain networks of the two firms are identically equal to zero, then the associated synergy can never be negative.

\section{Theorem 3}

If the total cost functions associated with the integration/merger links from node 0 to nodes $A$ and $B$ for each product are identically equal to zero, then the associated synergy, $\mathcal{S}^{T C}$, can never be negative.

Proof: We first note that the pre-integration supply chain optimization problem can be defined over the same expanded network as in Figure 2 but with the cross-shipment links extracted and with the paths defined from node 0 to the retail nodes. In addition, the total costs from node 0 to nodes $\mathrm{A}$ and $\mathrm{B}$ must all be equal to zero. Clearly, the total cost minimization solution to this problem yields the same total cost value as obtained for $T C^{0}$. We must now show that $T C^{0}-T C^{1} \geq 0$.

Assume not, that is, that $T C^{0}-T C^{1}<0$, then, clearly, we have not obtained an optimal solution to the post-integration problem, since, the new links need not be used, which would imply that $T C^{0}=T C^{1}$, which is a contradiction.

Another interpretation of this theorem is that, in the system-optimization context (assuming that the total cost functions remain the same as do the demands), the addition of new links can never make the total cost increase; this is in contrast to what may occur in the context of user-optimized networks, where the addition of a new link may make everyone worse-off in terms of user cost. This is the well-known Braess paradox (1968); see, also, Braess et al. (2005).

\section{Numerical Examples}

In this Section, we present numerical examples for which we compute the solutions to the supply chains both pre and post the integration, along with the associated total costs and synergies as defined in Section 3. The examples were solved using the modified projection 
method (see, e.g., Korpelevich (1977) and Nagurney (2009)) embedded with the equilibration algorithm (cf. Dafermos and Sparrow (1969) and Nagurney (1984)). The modified projection method is guaranteed to converge if the function that enters the variational inequality is monotone and Lipschitz continuous (provided that a solution exists). Both these assumptions are satisfied under the conditions imposed on the multiproduct total cost functions in Section 2 as well as by the total cost functions underlying the numerical examples below. Since we also assume that the feasible sets are non-empty, we are guaranteed that the modified projection method will converge to a solution of variational inequalities (7) and (13).

We implemented the computational procedure in FORTRAN and utilized a Unix system at the University of Massachusetts Amherst for the computations. The algorithm was considered to have converged when the absolute value of the difference between the computed values of the variables (the link flows; respectively, the Lagrange multipliers) at two successive iterations differed by no more than $10^{-5}$. In order to fully exploit the underlying network structure, we first converted the multiproduct supply chain networks, into single-product "extended" ones as discussed in Dafermos (1973) for multimodal/multiclass traffic networks. The link capacity constraints, which do not explicitly appear in the original traffic network models, were adapted accordingly. The modified projecion method yielded subproblems, at each iteration, in flow variables and in price variables. The former were computed using the equilibration algorithm of Dafermos and Sparrow (1969) and the latter were computed explicitly and in closed form.

For all the numerical examples, we assumed that each firm $i ; i=A, B$, was involved in the production, storage, and distribution of two products, and each firm had, prior to the integration/merger, two manufacturing plants, one distribution center, and supplied the products to two retail outlets.

After the integration of the two firms' supply chain networks, each retailer was indifferent as to which firm supplied the products and the integrated/merged firms could store the products at any of the two distribution centers and could supply any of the four retailers. Figure 3 depicts the pre-integration supply chain network(s), whereas Figure 4 depicts the post-integration supply chain network for the numerical examples.

For all the examples, we assumed that the pre-integration total cost functions and the post-integration total cost functions were nonlinear (quadratic), of the form:

$$
\hat{c}_{a}^{j}\left(f_{a}^{1}, f_{a}^{2}\right)=\sum_{l=1}^{2} g_{a}^{j l} f_{a}^{j} f_{a}^{l}+h_{a}^{j} f_{a}^{j}, \quad \forall a \in L^{0}, \forall a \in L^{1} ; \quad j=1,2,
$$

with convexity of the total cost functions being satisfied (except, where noted, for the top- 

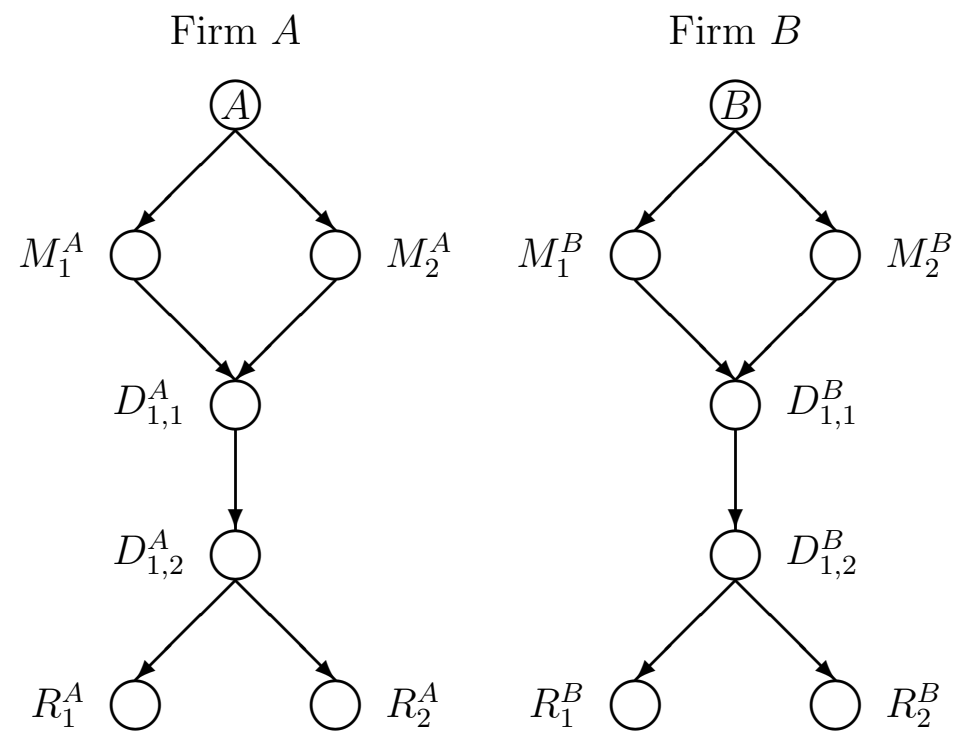

Figure 3: Pre-Integration Supply Chain Network Topology for the Numerical Examples most merger links from node 0). 


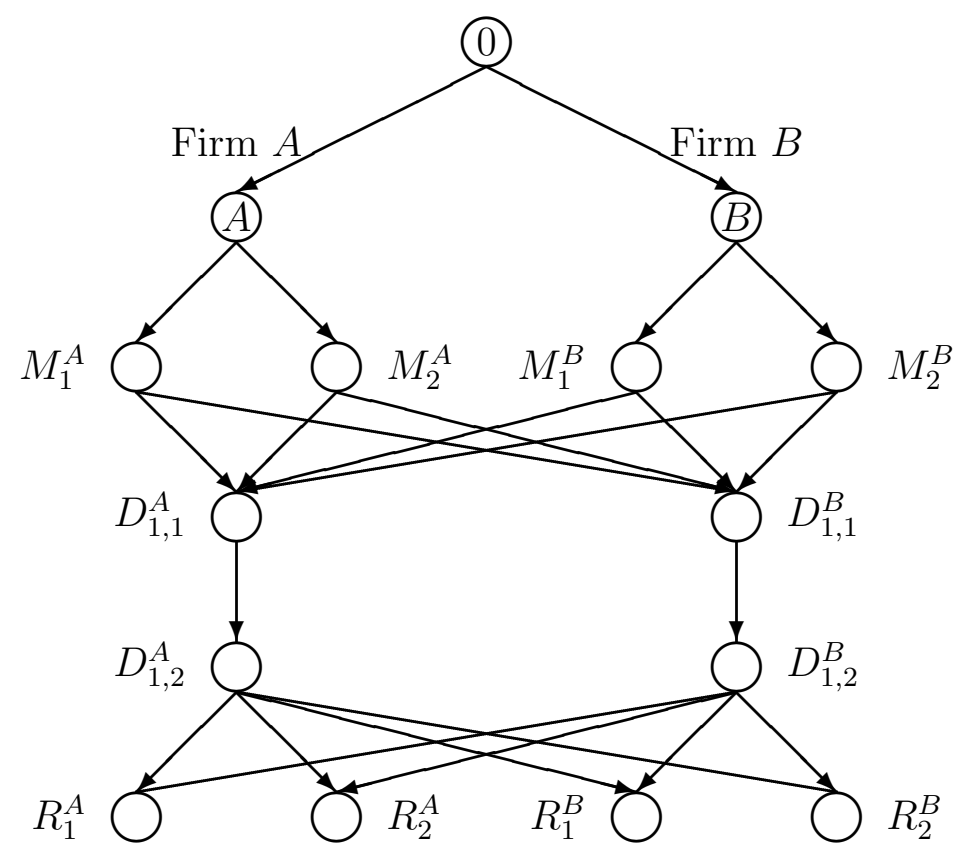

Figure 4: Post-Integration Supply Chain Network Topology for the Examples

\section{Example 1}

Example 1 served as the baseline for our computations. The Example 1 data are now described. The pre and post-integration total cost functions for products 1 and 2 are listed in Table 1. The links post-integration that join the node 0 with nodes $A$ and $B$ had associated total costs equal to zero for each product $j=1,2$, for Examples 1 through 3 . The demands at the retail outlets for Firm $A$ and Firm $B$ were set to 5 for each product. Hence, $d_{R_{k}^{i}}^{j}=5$ for $i=A, B ; j=1,2$, and $k=1,2$. The capacity on each link was set to 25 both pre and post integration, so that: $u_{a}=25$ for all links $a \in L^{0} ; a \in L^{1}$. The weights: $\alpha_{j}=1$ were set to 1 for both products $j=1,2$, both pre and post-integration; thus, we assumed that the products are equal in volume.

The pre-integration optimal solutions for the product flows for each product for Examples 1 through 3 are given in Table 2. We note that Example 1, pre-integration, was used as the basis from which variants post-integration were constructed, yielding Examples 2 and 3, as described below.

The post-integration optimal solutions are reported in Table 3 for product 1 and in Table 4 for product 2 .

Since none of the link flow capacities were reached, either pre- or post-integration, the vectors $\beta^{0 *}$ and $\beta^{1 *}$ had all their components equal to zero. The total cost, pre-merger, $T C^{0}=5,702.58$. The total cost, post-merger, $T C^{1}=4,240.86$. Please also refer to Table 5 for the total cost and synergy values for this example as well as for the next two examples. 
Table 1: Definition of Links and Associated Total Cost Functions for Example 1

\begin{tabular}{|c|c|c|c|c|}
\hline Link $a$ & From Node & To Node & $\hat{c}_{a}^{1}\left(f_{a}^{1}, f_{a}^{2}\right)$ & $\hat{c}_{a}^{2}\left(f_{a}^{1}, f_{a}^{2}\right)$ \\
\hline 1 & $A$ & $M_{1}^{A}$ & $1\left(f_{1}^{1}\right)^{2}+2 f_{1}^{2} f_{1}^{1}+11 f_{1}^{1}$ & $2\left(f_{1}^{2}\right)^{2}+2 f_{1}^{1} f_{1}^{2}+8 f_{1}^{2}$ \\
\hline 2 & $A$ & $M_{2}^{A}$ & $2\left(f_{2}^{1}\right)^{2}+2 f_{2}^{2} f_{2}^{1}+8 f_{2}^{1}$ & $1\left(f_{2}^{2}\right)^{2}+2 f_{2}^{1} f_{2}^{2}+6 f_{2}^{2}$ \\
\hline 3 & $M_{1}^{A}$ & $D_{1,1}^{A}$ & $3\left(f_{3}^{1}\right)^{2}+2.5 f_{3}^{2} f_{3}^{1}+7 f_{3}^{1}$ & $4\left(f_{3}^{2}\right)^{2}+2.5 f_{3}^{1} f_{3}^{2}+7 f_{3}^{2}$ \\
\hline 4 & $M_{2}^{A}$ & $D_{1,1}^{A}$ & $4\left(f_{4}^{1}\right)^{2}+1.5 f_{4}^{2} f_{4}^{1}+3 f_{4}^{1}$ & $3\left(f_{4}^{2}\right)^{2}+1.5 f_{4}^{1} f_{4}^{2}+11 f_{4}^{2}$ \\
\hline 5 & $D_{1,1}^{A}$ & $D_{1,2}^{A}$ & $1\left(f_{5}^{1}\right)^{2}+f_{5}^{2} f_{5}^{1}+6 f_{5}^{1}$ & $4\left(f_{5}^{2}\right)^{2}+f_{5}^{1} f_{5}^{2}+11 f_{5}^{2}$ \\
\hline 6 & $D_{1,2}^{A, 1}$ & $R_{1}^{A}$ & $3\left(f_{6}^{1}\right)^{2}+1.5 f_{6}^{2} f_{6}^{1}+4 f_{6}^{1}$ & $4\left(f_{6}^{2}\right)^{2}+1.5 f_{6}^{1} f_{6}^{2}+10 f_{6}^{2}$ \\
\hline 7 & $D_{1,2}^{A, 2}$ & $\stackrel{1}{A}$ & $4\left(f_{7}^{1}\right)^{2}+2 f_{7}^{2} f_{7}^{1}+7 f_{7}^{1}$ & $2\left(f_{7}^{2}\right)^{2}+2 f_{7}^{1} f_{7}^{2}+8 f_{7}^{2}$ \\
\hline 8 & $B$ & $M_{1}^{B}$ & $4\left(f_{8}^{1}\right)^{2}+3 f_{8}^{2} f_{8}^{1}+5 f_{8}^{1}$ & $4\left(f_{8}^{2}\right)^{2}+3 f_{8}^{1} f_{8}^{2}+6 f_{8}^{2}$ \\
\hline 9 & $B$ & $M_{2}^{B}$ & $1\left(f_{9}^{1}\right)^{2}+1.5 f_{9}^{2} f_{9}^{1}+4 f_{9}^{1}$ & $4\left(f_{9}^{2}\right)^{2}+1.5 f_{9}^{1} f_{9}^{2}+6 f_{9}^{2}$ \\
\hline 10 & $M_{1}^{B}$ & $D_{1,1}^{B}$ & $2\left(f_{10}^{1}\right)^{2}+3 f_{10}^{2} f_{10}^{1}+3.5 f_{10}^{1}$ & $3\left(f_{10}^{2}\right)^{2}+3 f_{10}^{1} f_{10}^{2}+4 f_{10}^{2}$ \\
\hline 11 & $M_{2}^{B}$ & $D_{1,1}^{B}$ & $1\left(f_{11}^{1}\right)^{2}+2.5 f_{11}^{2} f_{11}^{1}+4 f_{11}^{1}$ & $4\left(f_{11}^{2}\right)^{2}+2.5 f_{11}^{1} f_{11}^{2}+5 f_{11}^{2}$ \\
\hline 12 & $\stackrel{L}{B}$ & $\frac{1,1}{D_{1,2}^{B}}$ & $4\left(f_{12}^{1}\right)^{2}+3 f_{12}^{2} f_{12}^{1}+6 f_{12}^{1}$ & $2\left(f_{12}^{2}\right)^{2}+3 f_{12}^{1} f_{12}^{2}+5 f_{12}^{2}$ \\
\hline 13 & $\begin{array}{l}1,1 \\
D_{1,2}^{B}\end{array}$ & $R_{1}^{B}$ & $3\left(f_{13}^{1}\right)^{2}+3 f_{13}^{2} f_{13}^{1}+7 f_{13}^{1}$ & $4\left(f_{13}^{2}\right)^{2}+3 f_{13}^{1} f_{13}^{2}+10 f_{13}^{2}$ \\
\hline 14 & $D_{1,2}^{B, 2}$ & $R_{2}^{B}$ & $4\left(f_{14}^{1}\right)^{2}+.5 f_{14}^{2} f_{14}^{1}+4 f_{14}^{1}$ & $4\left(f_{14}^{2}\right)^{2}+.5 f_{14}^{1} f_{14}^{2}+12 f_{14}^{2}$ \\
\hline 15 & $M_{1}^{A}$ & $D_{1,1}^{B}$ & $4\left(f_{15}^{1}\right)^{2}+2 f_{15}^{2} f_{15}^{1}+6 f_{15}^{1}$ & $4\left(f_{15}^{2}\right)^{2}+2 f_{15}^{1} f_{15}^{2}+7 f_{15}^{2}$ \\
\hline 16 & $M_{2}^{A}$ & $D_{1,1}^{B}$ & $4\left(f_{16}^{1}\right)^{2}+2 f_{16}^{2} f_{16}^{1}+6 f_{16}^{1}$ & $3\left(f_{16}^{2}\right)^{2}+2 f_{16}^{1} f_{16}^{2}+7 f_{16}^{2}$ \\
\hline 17 & $M_{1}^{B}$ & $D_{1,1}^{A}$ & $1\left(f_{17}^{1}\right)^{2}+3.5 f_{17}^{2} f_{17}^{1}+4 f_{17}^{1}$ & $4\left(f_{17}^{2}\right)^{2}+3.5 f_{17}^{1} f_{17}^{2}+5 f_{17}^{2}$ \\
\hline 18 & $M_{2}^{B}$ & $\stackrel{1,1}{D_{1,1}^{A}}$ & $4\left(f_{18}^{1}\right)^{2}+3 f_{18}^{2} f_{18}^{1}+9 f_{18}^{1}$ & $4\left(f_{18}^{2}\right)^{2}+3 f_{18}^{1} f_{18}^{2}+7 f_{18}^{2}$ \\
\hline 19 & $\stackrel{2}{A}$ & $\frac{1,1}{R_{1}^{B}}$ & $4\left(f_{19}^{1}\right)^{2}+3.5 f_{19}^{2} f_{19}^{1}+7 f_{19}^{1}$ & $1\left(f_{19}^{2}\right)^{2}+3.5 f_{19}^{1} f_{19}^{2}+9 f_{19}^{2}$ \\
\hline 20 & $\frac{1,2}{D_{1,2}^{A}}$ & $R_{2}^{B}$ & $2\left(f_{20}^{1}\right)^{2}+3 f_{20}^{2} f_{20}^{1}+5 f_{20}^{1}$ & $4\left(f_{20}^{2}\right)^{2}+3 f_{20}^{1} f_{20}^{2}+6 f_{20}^{2}$ \\
\hline 21 & $\begin{array}{l}1,2 \\
D_{1,2}^{B}\end{array}$ & $\stackrel{2}{R_{1}^{A}}$ & $4\left(f_{21}^{1}\right)^{2}+2.5 f_{21}^{2} f_{21}^{1}+3 f_{21}^{1}$ & $3\left(f_{21}^{2}\right)^{2}+2.5 f_{21}^{1} f_{21}^{2}+9 f_{21}^{2}$ \\
\hline 22 & $\begin{array}{l}1,2 \\
D_{1,2}^{B}\end{array}$ & $R_{2}^{A}$ & $3\left(f_{22}^{1}\right)^{2}+2 f_{22}^{2} f_{22}^{1}+4 f_{22}^{1}$ & $4\left(f_{22}^{2}\right)^{2}+2 f_{22}^{1} f_{22}^{2}+3 f_{22}^{2}$ \\
\hline
\end{tabular}


Table 2: Pre-Integration Optimal Product Flow Solutions to Examples 1 Through 3

\begin{tabular}{|c|c|c|l|l|}
\hline Link $a$ & From Node & To Node & $f_{a}^{1 *}$ & $f_{a}^{2 *}$ \\
\hline 1 & $\mathrm{~A}$ & $M_{1}^{A}$ & 8.50 & .80 \\
\hline 2 & $\mathrm{~A}$ & $M_{2}^{A}$ & 1.50 & 9.20 \\
\hline 3 & $M_{1}^{A}$ & $D_{1,1}^{A}$ & 8.50 & .80 \\
\hline 4 & $M_{2}^{A}$ & $D_{1,1}^{A}$ & 1.50 & 9.20 \\
\hline 5 & $D_{1,1}^{A}$ & $D_{1,2}^{A}$ & 10.00 & 10.00 \\
\hline 6 & $D_{1,2}^{A}$ & $R_{1}^{A}$ & 5.00 & 5.00 \\
\hline 7 & $D_{1,2}^{A}$ & $R_{2}^{A}$ & 5.00 & 5.00 \\
\hline 8 & $\mathrm{~B}$ & $M_{1}^{B}$ & 0.00 & 8.03 \\
\hline 9 & $\mathrm{~B}$ & $M_{2}^{B}$ & 10.00 & 1.97 \\
\hline 10 & $M_{1}^{B}$ & $D_{1,1}^{B}$ & 0.00 & 8.03 \\
\hline 11 & $M_{2}^{B}$ & $D_{1,1}^{B}$ & 10.00 & 1.97 \\
\hline 12 & $D_{1,1}^{B}$ & $D_{1,2}^{B}$ & 10.00 & 10.00 \\
\hline 13 & $D_{1,2}^{B}$ & $R_{1}^{B}$ & 5.00 & 5.00 \\
\hline 14 & $D_{1,2}^{B}$ & $R_{2}^{B}$ & 5.00 & 5.00 \\
\hline
\end{tabular}

The synergy $\mathcal{S}^{T C}$ for the supply chain network integration for Example 1 was equal to $25.63 \%$.

It is interesting to note that, since the distribution center associated with the original Firm A has total storage costs that are lower for product 1, whereas Firm B's distribution center has lower costs associated with the storage of product 2, that Firm A's original distribution center, after the integration/merger, stores the majority of the volume of product 1, while the majority of the volume of product 2 is stored, post-integration, at Firm B's original distribution center. It is also interesting to note that, post-integration, the majority of the production of product 1 takes place in Firm B's original manufacturing plants, whereas the converse holds true for product 2. This example, hence, vividly illustrates the types of supply chain cost gains that can be achieved in the integration of multiproduct supply chains. 
Table 3: Post-Integration Optimal Flow Solutions to the Examples for Product 1

\begin{tabular}{|c|c|c|c|c|c|}
\hline Link $a$ & From Node & To Node & Ex. $1 f_{a}^{1 *}$ & Ex. $2 f_{a}^{1 *}$ & Ex. $3 f_{a}^{1 *}$ \\
\hline 1 & $A$ & $M_{1}^{A}$ & 5.94 & 0.76 & 5.36 \\
\hline 2 & $A$ & $M_{2}^{A}$ & 0.53 & 0.00 & 1.98 \\
\hline 3 & $M_{1}^{A}$ & $D_{1,1}^{A}$ & 5.94 & 0.00 & 5.36 \\
\hline 4 & $M_{2}^{A}$ & $D_{1,1}^{A}$ & 0.53 & 0.00 & 1.98 \\
\hline 5 & $D_{1,1}^{A}$ & $D_{1,2}^{A}$ & 18.27 & 19.24 & 17.34 \\
\hline 6 & $D_{1,2}^{A}$ & $R_{1}^{A}$ & 5.00 & 5.00 & 5.00 \\
\hline 7 & $D_{1,2}^{A}$ & $R_{2}^{A}$ & 3.27 & 4.24 & 4.27 \\
\hline 8 & $B$ & $M_{1}^{B}$ & 6.25 & 1.67 & 5.00 \\
\hline 9 & $B$ & $M_{2}^{B}$ & 7.29 & 17.57 & 7.66 \\
\hline 10 & $M_{1}^{B}$ & $D_{1,1}^{B}$ & 0.00 & 0.00 & 0.00 \\
\hline 11 & $M_{2}^{B}$ & $D_{1,1}^{B}$ & 1.73 & 0.00 & 2.66 \\
\hline 12 & $D_{1,1}^{B}$ & $D_{1,2}^{B}$ & 1.73 & 0.76 & 2.66 \\
\hline 13 & $D_{1,2}^{B}$ & $R_{1}^{B}$ & 0.00 & 0.00 & 0.00 \\
\hline 14 & $D_{1,2}^{B}$ & $R_{2}^{B}$ & 0.00 & 0.00 & 1.93 \\
\hline 15 & $M_{1}^{A}$ & $D_{1,1}^{B}$ & 0.00 & 0.76 & 0.00 \\
\hline 16 & $M_{2}^{A}$ & $D_{1,1}^{B}$ & 0.00 & 0.00 & 0.00 \\
\hline 17 & $M_{1}^{B}$ & $D_{1,1}^{A}$ & 6.25 & 1.67 & 5.00 \\
\hline 18 & $M_{2}^{B}$ & $D_{1,1}^{A}$ & 5.55 & 17.57 & 5.00 \\
\hline 19 & $D_{1,2}^{A}$ & $R_{1}^{B}$ & 5.00 & 5.00 & 5.00 \\
\hline 20 & $D_{1,2}^{A}$ & $R_{2}^{B}$ & 5.00 & 5.00 & 3.07 \\
\hline 21 & $D_{1,2}^{B}$ & $R_{1}^{A}$ & 0.00 & 0.00 & 0.00 \\
\hline 22 & $D_{1,2}^{B}$ & $R_{2}^{A}$ & 1.73 & 0.76 & 0.73 \\
\hline
\end{tabular}


Table 4: Post-Integration Optimal Flow Solutions to the Examples for Product 2

\begin{tabular}{|c|c|c|c|c|c|}
\hline Link $a$ & From Node & To Node & Ex. $1 f_{a}^{2 *}$ & Ex. $2 f_{a}^{2 *}$ & Ex. $3 f_{a}^{2 *}$ \\
\hline 1 & $A$ & $M_{1}^{A}$ & 3.44 & 4.66 & 5.00 \\
\hline 2 & $A$ & $M_{2}^{A}$ & 11.81 & 11.88 & 8.74 \\
\hline 3 & $M_{1}^{A}$ & $D_{1,1}^{A}$ & 0.00 & 0.88 & 0.00 \\
\hline 4 & $M_{2}^{A}$ & $D_{1,1}^{A}$ & 4.91 & 0.48 & 3.74 \\
\hline 5 & $D_{1,1}^{A}$ & $D_{1,2}^{A}$ & 4.91 & 4.82 & 3.74 \\
\hline 6 & $D_{1,2}^{A}$ & $R_{1}^{A}$ & 1.52 & 0.00 & 0.61 \\
\hline 7 & $D_{1,2}^{A}$ & $R_{2}^{A}$ & 2.58 & 0.00 & 1.20 \\
\hline 8 & $B$ & $M_{1}^{B}$ & 2.34 & 3.46 & 3.58 \\
\hline 9 & $B$ & $M_{2}^{B}$ & 2.42 & 0.00 & 2.68 \\
\hline 10 & $M_{1}^{B}$ & $D_{1,1}^{B}$ & 2.34 & 0.00 & 3.58 \\
\hline 11 & $M_{2}^{B}$ & $D_{1,1}^{B}$ & 2.42 & 0.00 & 2.68 \\
\hline 12 & $D_{1,1}^{B}$ & $D_{1,2}^{B}$ & 15.09 & 15.18 & 16.26 \\
\hline 13 & $D_{1,2}^{B}$ & $R_{1}^{B}$ & 4.88 & 2.72 & 5.00 \\
\hline 14 & $D_{1,2}^{B}$ & $R_{2}^{B}$ & 4.30 & 2.46 & 3.07 \\
\hline 15 & $M_{1}^{A}$ & $D_{1,1}^{B}$ & 3.44 & 3.78 & 5.00 \\
\hline 16 & $M_{2}^{A}$ & $D_{1,1}^{B}$ & 6.89 & 11.40 & 5.00 \\
\hline 17 & $M_{1}^{B}$ & $D_{1,1}^{A}$ & 0.00 & 3.46 & 0.00 \\
\hline 18 & $M_{2}^{B}$ & $D_{1,1}^{A}$ & 0.00 & 0.00 & 0.00 \\
\hline 19 & $D_{1,2}^{A}$ & $R_{1}^{B}$ & 0.12 & 2.28 & 0.00 \\
\hline 20 & $D_{1,2}^{A}$ & $R_{2}^{B}$ & 0.70 & 2.54 & 1.93 \\
\hline 21 & $D_{1,2}^{B}$ & $R_{1}^{A}$ & 3.48 & 5.00 & 4.39 \\
\hline 22 & $D_{1,2}^{B}$ & $R_{2}^{A}$ & 2.42 & 5.00 & 3.80 \\
\hline
\end{tabular}




\section{Example 2}

Example 2 was constructed from Example 1 but with the following modifications. We now considered an idealized situation in which we assumed that the total costs associated with the new integration links; see Table 1 (links 15 through 22) for each product were identically equal to zero.

Post-integration, the optimal flow for each product, for each firm, has now changed; see Table 3 and Table 4 . It is interesting to note that now the second manufacturing plant associated with the original Firm B produces the majority of product 1 but the majority of product 1 is still stored at the original distribution center of Firm A. Indeed, the zero costs associated with distribution between the original supply chain networks lead to further synergies as compared to those obtained for Example 1.

Since, again, none of the link flow capacities were reached, either pre- or post-integration, the vectors $\beta^{0 *}$ and $\beta^{1 *}$ had all their components equal to zero. The total cost, post-merger, $T C^{1}=2,570.27$. The synergy $\mathcal{S}^{T C}$ for the supply chain network integration for Example 2 was equal to $54.93 \%$. Observe that this obtained synergy is, in a sense, the maximum possible for this example since the total costs for both products on all the new links are all equal to zero.

\section{Example 3}

Example 3 was constructed from Example 2 but with the following modifications. We now assumed that the capacities associated with the links that had zero costs between the two original firms had their capacities reduced from 25 to 5 . The computed optimal flow solutions are given in Table 3 for product 1 and in Table 4 for product 2 .

We now also provide the computed vector of Lagrange multipliers $\beta^{1 *}$. All terms were equal to zero except those for links 15 through 20 since the sum of the corresponding product flows on each of these links was equal to the imposed capacity of 5 . In particular, we now had: $\beta_{15}^{*}=40.82, \beta_{16}^{*}=59.79, \beta_{17}^{*}=14.35, \beta_{18}^{*}=53.59, \beta_{19}^{*}=79.95$, and $\beta_{20}^{*}=68.39$.

The total cost, post-merger, was now $T C^{1}=3,452.34$. The synergy $\mathcal{S}^{T C}$ for the supply chain network integration for Example 3 was equal to $39.46 \%$. Hence, even with substantially lower capacities on the new links, given the zero costs, the synergy associated with the supply chain network integration in Example 3 was quite high, although not as high as obtained in Example 2.

Firm B's original distribution center now stores more of product 1 and 2 than it did 
Table 5: Total Costs and Synergy Values for the Examples

\begin{tabular}{|c|c|c|c|}
\hline Measure & Example 1 & Example 2 & Example 3 \\
\hline Pre-Integration $T C^{0}$ & $5,702.58$ & $5,702.58$ & $5,702.58$ \\
\hline Post-Integration $T C^{1}$ & $4,240.86$ & $2,570.27$ & $3,452.34$ \\
\hline Synergy Calculations $\mathcal{S}^{T C}$ & $25.63 \%$ & $54.93 \%$ & $39.46 \%$ \\
\hline
\end{tabular}

in Example 2 (post-integration). Also, because of capacity reductions associated with the cross-shipment links there is a notable reduction in the volume of shipment of product 1 from the second manufacturing plant of Firm B to Firm A's original distribution center and in the shipment of product 2 from Firm A's original second manufacturing plant to Firm B's original distribution center.

\section{Additional Computations/Examples}

We then proceeded to ask the following question: assuming that the links, post-merger, joining node 0 to nodes $A$ and $B$ no longer had zero associated total cost for each product but, rather, reflected a cost associated with merging the two firms. We further assumed that the cost (cf. (15)) was linear and of the specific form given by

$$
\hat{c}_{a}^{j}=h_{a}^{j} f_{a}^{j}=h f_{a}^{j}, \quad j=1,2
$$

for the upper-most links (cf. Figure 4). Hence, we assumed that all the $h_{a}^{j}$ terms were identical and equal to an $h$. At what value would the synergy then for Examples 1, 2, and 3 become negative? Through computational experiments we were able to determine these values. In the case of Example 1, if $h=36.52$, then the synergy value would be approximately equal to zero since the new total cost would be approximately equal to $T C^{0}=5,702.58$. For any value larger than the above $h$, one would obtain negative synergy. This has clear implications for mergers in terms of supply chain network integration and demonstrates that the total costs associated with the integration/merger itself have to be carefully weighed against the cost benefits associated with the integrated supply chain activities. In the case of Example 2, the $h$ value was approximately equal to 78.3. A higher value than this $h$ for each such merger link would result in the total cost exceeding $T C^{0}$ and, hence, negative synergy would result.

Finally, for completeness, we also determined the corresponding $h$ in the case of Example 3 and found the value to be $h=78.3$, as in Example 2 . 


\section{Summary and Conclusions}

In this paper, we developed multiproduct supply chain network models, which allow one to evaluate the total costs associated with manufacturing/production, storage, and distribution of firms' supply chains both pre and post-integration. Such horizontal integrations can take place, for example, in the context of mergers and acquisitions, an activity which has garnered much interest and momentum recently. The model(s) utilize a system-optimization perspective and allow for explicit upper bounds on the various links associated with manufacturing, storage, and distribution. The models are formulated and solved as variational inequality problems.

In addition, we utilized a proposed multiproduct synergy measure to identify the potential cost gains associated with such horizontal supply chain network integrations. We proved that, in the case of zero "merging" costs, that the associated synergy can never be negative. We computed solutions to several numerical examples for which we determine the optimal product flows and Lagrange multipliers/shadow prices associated with the capacity constraints both before and after the integration. The computational approach allows one to explore many issues regarding supply chain network integration and to effectively ascertain the synergies prior to any implementation of a potential merger. In addition, we determined, computationally, for several examples, what identical linear costs would yield zero synergy, with higher values resulting in negative synergy.

There are numerous questions that remain and that will be considered for future research. It would be interesting to develop competitive variants of the models in a game theoretic context and to also explore elastic demands. Also, this paper does consider the time dimension in that it models the supply chain networks before and after the proposed merger and, hence, it considers two distinct point in time. For certain applications it may be useful to have a more detailed time discretization with accompanying network structure. Finally, it would be very interesting to explicitly incorporate the risks associated with supply chain network integration within our framework.

\section{Acknowledgments}

This research was supported by the John F. Smith Memorial Fund at the Isenberg School of Management. This support is gratefully acknowledged. The authors would also like to thank Professor June Dong for helpful discussions.

The authors acknowledge the helpful comments and suggestions received during the review process. 


\section{References}

Bazaraa, M.S., Sherali, H.D., Shetty, C.M., 1993. Nonlinear programming: Theory and algorithms (2nd edition). John Wiley \& Sons, New York.

Beckmann, M.J., McGuire, C.B., Winsten, C.B., 1956. Studies in the economics of transportation. Yale University Press, New Haven, Connecticut.

Bernard, A.B., Redding, S.J., Schott, P.K., 2006. Multi-product firms and product switching. Working paper, Dartmouth College, Hanover, New Hampshire;

http://mba.tuck.dartmouth.edu/pages/faculty/andrew.bernard/pswitch.pdf

Bertsekas, D.P., Tsitsiklis, J.N., 1989. Parallel and distributed computation - numerical methods. Prentice Hall, Englewood Cliffs, New Jersey.

Braess, D., 1968. Uber ein paradoxon der verkehrsplanung. Unternehmenforschung 12, $258-268$

Braess, D., Nagurney, A., Wakolbinger, T., 2005. On a paradox of traffic planning (translation of the Braess (1968) article from German). Transportation Science 39, 443-445.

Byrne, P.M. January 1, 2007. Unleashing supply chain value in mergers and acquisitions. Logistics Management, 20-20.

Chang, P.C., 1988. A measure of the synergy in mergers under a competitive market for corporate control. Atlantic Economic Journal 16, 59-62.

Cheng, T.C.E, Wu, Y.N., 2006. A multiproduct, multicriterion supply-demand network equilibrium model. Operations Research 54, 544-554.

Dafermos, S.C., 1973. The traffic assignment problem for multiclass-user transportation networks. Transportation Science 6, 73-87.

Dafermos, S.C., Sparrow, F.T., 1969. The traffic assignment problem for a general network. Journal of Research of the National Bureau of Standards 73B, 91-118.

Davis, D.D., Wilson, B.J., 2006. Equilibrium price dispersion, mergers and synergies: An experimental investigation of differentiated product competition. International Journal of the Economics of Business 13, 169-194.

Dong, J., Zhang, D., Nagurney, A., 2004. A supply chain network equilibrium model with random demands. European Journal of Operational Research 156, 194-212. 
Eccles, R.G., Lanes, K.L., Wilson, T.C., 1999. Are you paying too much for that acquisition? Harvard Business Review 77, 136-146.

Korpelevich, G.M., 1977. The extragradient method for finding saddle points and other problems. Matekon 13, 35-49.

Langabeer, J., Seifert, D., 2003. Supply chain integration: The key to merger success. Supply Chain Management Review 7, 58-64.

Min, H., Zhou, G., 2002. Supply chain modeling: Past, present and future. Computers and Industrial Engineering 43, 231-249.

Nagurney, A., 1984. Computational comparisons of algorithms for general asymmetric traffic equilibrium problems with fixed and elastic demands. Transportation Research $B$ 18, 469485.

Nagurney, A., 1999. Network economics: a variational inequality approach (2nd edition). Kluwer Academic Publishers, Dordrecht, The Netherlands.

Nagurney, A., 2006a. Supply chain network economics: Dynamics of prices, flows and profits. Edward Elgar Publishing, Cheltenham, England.

Nagurney, A., 2006b. On the relationship between supply chain and transportation network equilibria: A supernetwork equivalence with computations. Transportation Research E 42, 293-316.

Nagurney, A., 2009. A system-optimization perspective for supply chain integration: The horizontal merger case. Transportation Research E 45, 1-15.

Soylu, A., Oru, C., Turkay, M., Fujita, K., Asakura, T., 2006. Synergy analysis of collaborative supply chain management in energy systems using multi-period MILP. European Journal of Operational Research 174, 387-403.

Wang, Z., Zhang, F., Wang, Z., 2007. Research of return supply chain supernetwork model based on variational inequalities. Proceedings of the IEEE International Conference on Automation and Logistics 25-30, Jinan, China.

Wong, G., October 10, 2007. After credit crisis, New forces drive deals. CNNMoney.com.

$\mathrm{Xu}$, S., 2007. Supply chain synergy in mergers and acquisitions: Strategies, models and key factors, PhD dissertation, University of Massachusetts, Amherst, Massachusetts. 